\title{
Radiation Pattern Analysis of Antenna Systems for MIMO and Diversity Configurations
}

\author{
O. Klemp and H. Eul \\ University of Hannover, Department of High Frequency Technology and Radio Systems, Hannover, Germany
}

\begin{abstract}
Multiple-input multiple-output (MIMO) antenna systems and antenna configurations for wideband multimode diversity rank among the emerging key technologies in next generation wireless communication systems. The analysis of such transmission systems usually neglects the influences of real antenna radiation characteristics as well as the influences of mutual coupling in a multielement antenna arrangement. Nevertheless, to achieve a detailed description of diversity gain and channel capacity by using several transmit- and receive antennas in a wireless link, it is essential to take all those effects into account. The expansion of the radiation fields in terms of spherical eigenmodes allows an analytical description of the antenna radiation characteristics and accounts for all the coupling effects in multielement antenna configurations. Therefore the radiation pattern analysis by spherical eigenmode expansion provides an efficient alternative to establish an analytical approach in the calculation of envelope correlation or channel capacity.
\end{abstract}

\section{Introduction}

The spherical mode expansion (SME) is a well known technique in terms of the characterization of a various number of applications of classical electromagnetic scattering and radiation problems. Numerous authors such as Stratton (1941) and Werner and Mittra (2000) have presented a generalized theory employing spherical mode analysis and adopted this method to study a multiplicity of wave propagation problems. Applications of the spherical mode expansion have been applied e.g. to the numerical analysis of Cassegrainianfed paraboloids in Potter (1967), the investigation of multiple scattering of electromagnetic waves in Bruning and Lo (1971), the computation of antenna radiation patterns of arbitrary wire antennas in Chen and Simpson (1991b,a) and the modeling of base station antenna equipment in Adane et al. (2002). Furthermore the application of spherical mode expansion to perform a transformation between near- and farfield related field quantities as described in Ludwig (1971) is a fundamental basis for todays techniques of spherical antenna near field measurement.

Correspondence to: O. Klemp

(klemp@hft.uni-hannover.de)
In this article the analysis of the radiation behavior using spherical eigenmodes will be applied to the characterization of the diversity performance of multielement antenna configurations in terms of interelement correlation. Therefore we present a modified technique of spherical mode expansion as in Chen et al. (1992) for the analysis of arbitrary antennas and multielement antennas. Based upon a suitable statistical model, e.g. provided in Waldschmidt and Wiesbeck (2004) to include the spatial properties of a multipath propagation scenario, a closed form representation for antenna correlation will be derived.

The article is organized as follows, Sect. 2 reviews the fundamental properties of the spherical mode expansion technique with respect to the electric- and magnetic vector potential for a given elementary source distribution. Section 3 derives a generalized representation of antenna radiation pattern due to the Fraunhofer approximation of the Green's radiation function. Subsequently the modal representation of antenna radiation patterns is used in Sect. 4 to specify the power correlation between interactive antenna elements in a diversity configuration to establish a measure for their diversity performance. The presented analysis is adopted to an array of resonant $\lambda / 2$ dipoles given in Sects. 5.1 and 5.2 and a configuration of multi-port wideband logarithmically periodic antennas in Sect. 6. The latter analysis will be completed by results derived from spherical near-field measurement. Section 7 concludes this article.

\section{Modal Solutions of Vector Potentials}

The spherical mode expansion as given in this section will be used to accomplish the transformation between a known distribution of electric and magnetic currents (e.g. on the metalization surfaces of an antenna structure) and the resulting electromagnetic field distribution in the field region, which is considered to be source-free. In Ludwig (1971) this transformation technique was applied to derive a generalized mapping rule between field quantities in different coordinate systems. Therefore in SME analysis a separation of coordinate systems for the given source-region and the requested fieldregion is required. For a given volume $V$ containing electricand magnetic current densities $\boldsymbol{J}$ and $\boldsymbol{J}_{\mathrm{m}}$ as shown in Fig. 1 the magnetic- and electric vector potentials $\boldsymbol{A}$ and $\boldsymbol{F}$ can be 


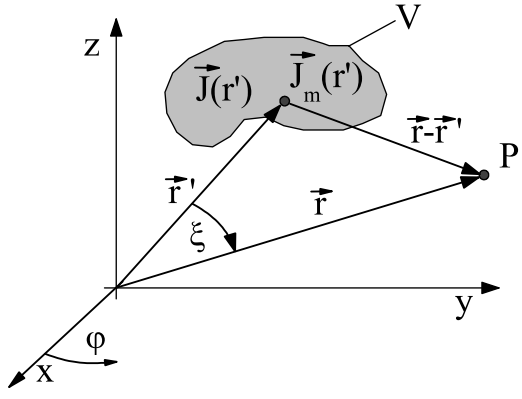

Fig. 1. Distribution of electric and magnetic current densities $\boldsymbol{J}$ and $J_{\mathrm{m}}$ in volume $V$.

represented using Chen et al. (1992) as follows:

$$
\begin{aligned}
& \boldsymbol{A}(\boldsymbol{r})=\frac{1}{4 \pi} \iiint_{\left(V^{\prime}\right)} \frac{\boldsymbol{J}\left(\boldsymbol{r}^{\prime}\right)}{\left|\boldsymbol{r}-\boldsymbol{r}^{\prime}\right|} e^{-j k\left|\boldsymbol{r}-\boldsymbol{r}^{\prime}\right|} d V^{\prime} \\
& \boldsymbol{F}(\boldsymbol{r})=\frac{1}{4 \pi} \iiint_{\left(V^{\prime}\right)} \frac{\boldsymbol{J}_{m}\left(\boldsymbol{r}^{\prime}\right)}{\left|\boldsymbol{r}-\boldsymbol{r}^{\prime}\right|} e^{-j k\left|\boldsymbol{r}-\boldsymbol{r}^{\prime}\right|} d V^{\prime} .
\end{aligned}
$$

In Eq. (1) $k=2 \pi \sqrt{\epsilon \mu} f$ denotes the wave number of the electromagnetic field, where $\epsilon$ represents the permittivity of free space and $\mu$ the respective permeability. Harmonic time dependence will be assumed, so that $f$ is equivalent to the operational frequency of any elementary source considered in the volume $V$. The vector $\boldsymbol{r}$ represents the distance between the origin and the point of observation and $\boldsymbol{r}^{\prime}$ gives the displacement of the source point in relation to the origin. Therefore $\boldsymbol{A}$ and $\boldsymbol{F}$ represent a solution to the vector Helmholtz equation of the total electric field $\boldsymbol{E}$ and magnetic field $\boldsymbol{H}$. Subsequently, the analysis will be restricted to the distribution of electric current densities, where the total radiation field is exclusively given in terms of the magnetic vector potential. Using the theorem of dual fields in electrodynamics an arbitrary distribution of the magnetic current density $\boldsymbol{J}_{\mathrm{m}}$ can be related to an electric current density $\boldsymbol{J}$, for this purpose (1) represents a complete solution of the radiation problem. Provided the field solution of the source distribution is considered in terms of the spherical coordinate system $(r, \vartheta, \varphi)$, the Green's function may be represented as in Chen and Simpson (1991a) using the zero-order spherical Hankel function $h_{0}^{(2)}$ of the second kind as given in Eq. (2) with $R=\left|\boldsymbol{r}-\boldsymbol{r}^{\prime}\right|$ :

$\frac{e^{-j k R}}{R}=-j k h_{0}^{(2)}(k R)$

For the validity of Eq. (2), the case where $\boldsymbol{r}>\boldsymbol{r}^{\prime}$ has to be considered, where the antennas will be analyzed in the transmit case. Applying the principle of reciprocity in antenna analysis, the receive case is implicitly given in Eq. (2). To derive a modal representation of the electromagnetic field, first of all Eq. (2) can be re-formulated in terms of an infinite series as given in Eq. (3). Using $n$.-order spherical Bessel functions $j_{\mathrm{n}}\left(k r^{\prime}\right)$ in terms of the source-point dependence, $n$.-order spherical Hankel functions $h_{\mathrm{n}}^{(2)}(k r)$ of the second kind for the dependence of the observation-point and Legendre polynomials $P_{\mathrm{n}}(\cos \xi)$ of degree $n$, Eq. (2) is the first step to derive a separation of source- and field region.

$h_{0}^{(2)}(k R)=\sum_{n=0}^{\infty}(2 n+1) j_{\mathrm{n}}\left(k r^{\prime}\right) h_{\mathrm{n}}^{(2)}(k r) P_{\mathrm{n}}(\cos \xi)$.

Using the addition theorem for Legendre polynomials as in Abramovitz and Stegun (1972),

$$
\begin{aligned}
P_{\mathrm{n}}(\cos \xi) & =\sum_{m=0}^{n} \epsilon_{\mathrm{m}} \frac{(n-m) !}{(n+m) !} \\
& \cdot P_{\mathrm{n}}^{\mathrm{m}}(\cos \vartheta) P_{\mathrm{n}}^{\mathrm{m}}\left(\cos \vartheta^{\prime}\right) \cos \left(m\left(\varphi-\varphi^{\prime}\right)\right)
\end{aligned}
$$

Eq. (5) gives a generalized solution to the magnetic vector potential. $P_{\mathrm{n}}^{\mathrm{m}}(\cos \vartheta)$ denotes the associated Legendre function of degree $n$ and order $m, \epsilon_{\mathrm{m}}$ specifies Neumann's number ( 1 for $m=0,2$ for $m>0)$.

$$
\begin{aligned}
& \boldsymbol{A}(\boldsymbol{r})= \\
& -j k \sum_{n=0}^{\infty} \sum_{m=0}^{n} \boldsymbol{a}_{\mathrm{n}}^{\mathrm{m}^{\prime}} N_{\mathrm{n}}^{\mathrm{m}} h_{\mathrm{n}}^{(2)}(k r) P_{\mathrm{n}}^{\mathrm{m}}(\cos \vartheta) \cos (m \varphi) \\
& -j k \sum_{n=0}^{\infty} \sum_{m=0}^{n} \boldsymbol{b}_{\mathrm{n}}^{\mathrm{m}^{\prime}} N_{\mathrm{n}}^{\mathrm{m}} h_{\mathrm{n}}^{(2)}(k r) P_{\mathrm{n}}^{\mathrm{m}}(\cos \vartheta) \sin (m \varphi) .
\end{aligned}
$$

In Eq. (5) the response to the given electric current distribution is separated with respect to source point- and field point dependent quantities using the normalization $N_{\mathrm{n}}^{\mathrm{m}}$ given in Eq. (6).

$N_{\mathrm{n}}^{\mathrm{m}}=\sqrt{\epsilon_{\mathrm{m}} \frac{(2 n+1)(n-m) !}{4 \pi(n+m) !}}$

Orthogonality between the axes of the spherical coordinate systems approves a separation of functional quantities that are solely related to a single coordinate direction. Therefore the sources of the radiation fields (given in primed coordinates) may be analyzed independently from the respective electromagnetic fields at the field-points. Any source dependence is given in terms of the vector expansion coefficients $\boldsymbol{a}_{\mathrm{n}}^{\mathrm{m}^{\prime}}$ and $\boldsymbol{b}_{\mathrm{n}}^{\mathrm{m}^{\prime}}$ of the field expansion as given in Eqs. (7) and (8).

$$
\begin{aligned}
& \boldsymbol{a}_{\mathrm{n}}^{\mathrm{m}^{\prime}}=\iiint_{\left(V^{\prime}\right)} N_{\mathrm{n}}^{\mathrm{m}} \boldsymbol{J}\left(\boldsymbol{r}^{\prime}\right) j_{\mathrm{n}}\left(k r^{\prime}\right) \\
& \cdot P_{\mathrm{n}}^{\mathrm{m}}\left(\cos \vartheta^{\prime}\right) \cos \left(m \varphi^{\prime}\right) d V^{\prime} \\
& \boldsymbol{b}_{\mathrm{n}}^{\mathrm{m}^{\prime}}=\iiint_{\left(V^{\prime}\right)} N_{\mathrm{n}}^{\mathrm{m}} \boldsymbol{J}\left(\boldsymbol{r}^{\prime}\right) j_{\mathrm{n}}\left(k r^{\prime}\right) \\
& \cdot P_{\mathrm{n}}^{\mathrm{m}}\left(\cos \vartheta^{\prime}\right) \sin \left(m \varphi^{\prime}\right) d V^{\prime}
\end{aligned}
$$

Therefore, Eqs. (7) and (8) denote a generalized description of all considered field sources in volume $V$ in terms of elementary spherical wave contributions. 


\section{Antenna Radiation pattern analysis}

Considering the radiation behavior of antenna elements usually the far-field approximation by Fraunhofer is applied. This approximation given in Eq. (9) neglects differences in amplitude of all incoming elementary waves at a certain far field distance $r$ so that the response to all field sources leads to a wave propagation with planar amplitude distribution. Thus effects of interference as a consequence of spatial separation between the field sources lead to a maximum phase deviation of $\pi / 8$ for all field points that adhere to the far field conditions.

$|\boldsymbol{r}|>8 \frac{a^{2}}{\lambda}$.

In Eq. (9) $a$ denotes the radius of a sphere that completely encloses the region of distributed source points. As a consequence of the Fraunhofer approximation, Eq. (3) can be simplified with respect to the spherical Hankel function:

$$
\lim _{|\boldsymbol{r}|>8 \frac{a^{2}}{\lambda}} h_{\mathrm{n}}^{(2)}(k r)=\frac{j^{n+1}}{k r} e^{-j k r} .
$$

In spherical coordinates $(\vartheta, \varphi)$, the vector potentials $\boldsymbol{A}$ or $\boldsymbol{F}$ may be directly mapped to the respective field quantities $\boldsymbol{E}$ or $\boldsymbol{H}$. Under the terms of an exclusive distribution of electric currents $\boldsymbol{J}$ in the considered source region and a field point adhering to the far field condition by Fraunhofer, Eq. (11) represents the complete solution of the electric field in terms of the spherical components $E_{\vartheta}$ and $E_{\varphi}$.

$\boldsymbol{E}(\vartheta, \varphi)=\left[\begin{array}{l}E_{\vartheta} \\ E_{\varphi}\end{array}\right]=-j k Z\left[\begin{array}{l}A_{\vartheta} \\ A_{\varphi}\end{array}\right]$.

In Eq. (11) $Z$ denotes the value of characteristic free-space impedance given by $Z=120 \pi \Omega$.

$$
\begin{aligned}
\boldsymbol{E}(\vartheta, \varphi)= & \frac{e^{-j k r}}{r} \sum_{n=0}^{\infty} \sum_{m=0}^{n} N_{\mathrm{n}}^{\mathrm{m}}\left\{\boldsymbol{a}_{\mathrm{n}}^{\mathrm{m}} \cos (m \varphi)\right. \\
& \left.+\boldsymbol{b}_{\mathrm{n}}^{\mathrm{m}} \sin (m \varphi)\right\} j^{\mathrm{n}} P_{\mathrm{n}}^{\mathrm{m}}(\cos \vartheta) .
\end{aligned}
$$

Therefore, Eq. (12) represents the far-field solution of the electromagnetic field for a transmit antenna in terms of a series representation of spherical harmonics. The respective source distribution is formulated in terms of an electric current distribution in accordance to Fig. 1 and Eq. (1). Due to the limitation of far field conditions, the spatial orientation of transverse electromagnetic field components becomes orthogonal in the far field plane, that again exhibits a normal orientation to the radial direction of wave propagation at any point of observation. Therefore the respective dependence of electrical field $\boldsymbol{E}$ and magnetic vector potential $\boldsymbol{A}$ is specified by Eq. (11) for the special case of spherical coordinates. Applying Eq. (12) a generalized description of antenna radiation pattern is derived by means of an infinite series expansion implying spherical harmonics. For this reason Eq. (13) includes the spatial properties of antenna radiation in terms of the vector antenna radiation pattern $\boldsymbol{C}(\vartheta, \varphi)$.

$\boldsymbol{E}(\vartheta, \varphi)=\frac{e^{-j k r}}{r} \boldsymbol{C}(\vartheta, \varphi)$
The vector expansion coefficients of the field approach, $\boldsymbol{a}_{\mathrm{n}}^{\mathrm{m}}$ and $\boldsymbol{b}_{\mathrm{n}}^{\mathrm{m}}$ in terms of the corresponding antenna radiation pattern, are related to the expansion coefficients of the magnetic vector potential $\boldsymbol{a}_{\mathrm{n}}^{\mathrm{m}^{\prime}}$ and $\boldsymbol{b}_{\mathrm{n}}^{\mathrm{m}^{\prime}}$ employing Eqs. (9) and (11) as follows:

$\left\{\begin{array}{l}a_{\mathrm{n}, \vartheta}^{\mathrm{m}} \\ a_{\mathrm{n}, \varphi}^{\mathrm{m}}\end{array}\right\}=-j k Z\left\{\begin{array}{l}a_{\mathrm{n}, \vartheta}^{\mathrm{m}^{\prime}} \\ a_{\mathrm{n}, \varphi}^{\mathrm{m}^{\prime}}\end{array}\right\}$,

and

$\left\{\begin{array}{l}b_{n, \vartheta}^{m} \\ b_{\mathrm{n}, \varphi}^{\mathrm{m}}\end{array}\right\}=-j k Z\left\{\begin{array}{l}b_{\mathrm{n}, \vartheta}^{\mathrm{m}^{\prime}} \\ b_{\mathrm{n}, \varphi}^{\mathrm{m}^{\prime}}\end{array}\right\}$

The normalized eigensolutions to the vector Helmholtz equation are given in terms of the normalized spherical harmonics $Y_{\mathrm{nm}}^{\mathrm{e}}(\vartheta, \varphi)$ and $Y_{\mathrm{nm}}^{\mathrm{o}}(\vartheta, \varphi)$ as given in Stratton (1941) due to:

$\left\{\begin{array}{c}Y_{\mathrm{nm}}^{\mathrm{e}}(\vartheta, \varphi) \\ Y_{\mathrm{nm}}^{\mathrm{o}}(\vartheta, \varphi)\end{array}\right\}=N_{\mathrm{n}}^{m} P_{\mathrm{n}}^{\mathrm{m}}(\cos \vartheta)\left\{\begin{array}{c}\cos (m \varphi) \\ \sin (m \varphi)\end{array}\right\}$.

With respect to the orthogonality property between spherical harmonics of different degree $n$ or order $m$ as shown in Werner and Mittra (2000), the field expansion as given in Eq. (12) represents a complete solution to the radiation behavior of an arbitrary source configuration represented in terms of vector expansion coefficients $\boldsymbol{a}_{\mathrm{n}}^{\mathrm{m}}$ and $\boldsymbol{b}_{\mathrm{n}}^{\mathrm{m}}$.

For practical considerations the infinite upper summation bound of Eq. (12) has to be limited to a finite value $N$. Impairments resulting from the limitation of the infinite summation can be isolated with respect to the requirement of approximative field convergence e.g. given by Narasimhan et al. (1985). Therefore modal indices greater $N_{\min }=k a$ are regarded to have little significance to the field composition, where $a$ denotes the minimum radius of a sphere that completely encloses the source antenna. A numerical study of the convergence behavior of the field expansion is given e.g. in Chen and Simpson (1991a) and Chen et al. (1992).

The vector coefficients of the field expansion can be determined according to Witte et al. (2003) by methods of numerical point matching as given in Hafner (1990). As described in Ludwig (1971) the matching procedure will be applied on the surface on which the fields are known. Subsequently the far field sphere is regarded to be the surface where the field quantities are given in terms of the far field components of antenna radiation pattern. In order to determine the requested modal weights, an equiangular grid is placed upon the far field sphere providing constant angular steps of $\Delta \varphi=2 \pi / \mathrm{P}$ and $\Delta \vartheta=\pi / \mathrm{Q} . P$ and $Q$ correspond to the number of field points in the directions of azimuth and elevation, respectively. Antenna radiation patterns are derived from numerical analysis employing the FEM.

\section{Computation of Multielement Antenna Correlation}

Antenna diversity reception is an effective technique for suppressing multipath fading in a mobile antenna system. Diversity reception attains effective fading mitigation by the synthesis, selection, and switching of several received signals 


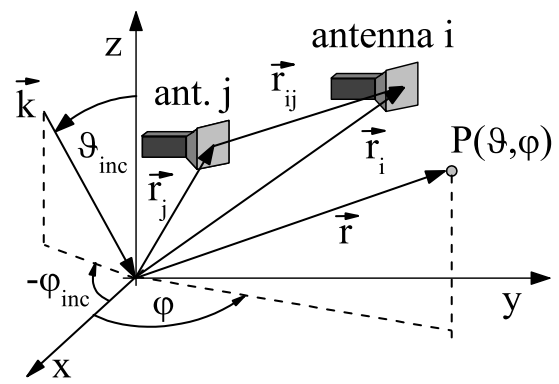

Fig. 2. Two-element antenna configuration in free space with antennas $i$ and $j$.

that are correlated to the smallest possible extent. In the case of antenna diversity, reduction of the correlation coefficient between the receiving antenna branches leads to an enhanced diversity effect. Correlation analysis may be performed using a representation of transmission line parameters like input impedances and scattering parameters at the individual diversity branches as demonstrated in Kildal and Rosengren (2003) and Derneryd and Kristensson (2004). The derived correlation coefficients do not account for the spatial properties of the transmit channel, where the receive antenna array is applied, but inherently assume a uniform distribution of the angle of arrival (AOA). Essentially, interelement correlation coefficients in a multielement antenna configuration are strongly affected by the properties of the considered multipath propagation environment as will be shown in the following. In Jakes (1974) the spatial properties of a multielement diversity antenna configuration may be acquired by applying two-dimensional probability distributions $p(\vartheta, \varphi)$ in terms of the AOA distribution of the incoming electromagnetic field in elevation- and azimuth direction. Examples for correlation analysis in multielement antennas may be found in Tsunekawa and Kagoshima (1990), Svantesson (2002) and Waldschmidt and Wiesbeck (2004).

$\rho_{P_{\mathrm{i}, \mathrm{j}}} \simeq\left|\rho_{\mathrm{i}, \mathrm{j}}\right|^{2}=\left|\frac{R_{\mathrm{i}, \mathrm{j}}}{\sqrt{\sigma_{\mathrm{i}}^{2} \sigma_{\mathrm{j}}^{2}}}\right|^{2}$.

Subsequently, the relation between power correlation coefficient $\rho_{P_{\mathrm{i}, \mathrm{j}}}$ and complex correlation coefficient $\rho_{\mathrm{i}, \mathrm{j}}$ as given in Eq. (17) between antenna elements $i$ and $j$ will be considered. The power correlation therefore depends on the covariance $R_{\mathrm{i}, \mathrm{j}}$ between the two antenna elements and the variances $\sigma_{i}^{2}$ and $\sigma_{j}^{2}$ that are detected at the respective diversity branches. Figure 2 depicts the corresponding configuration of a two-element antenna in receive mode. The distance vector $\boldsymbol{r}_{\mathrm{ij}}$ accounts for the spatial separation of antennas $i$ and $j$. As shown in Leifer (2002) the spatial separation between the antenna elements results in a receive gain due to spatial diversity. The direction of plane waves impinging at a direction $\vartheta_{\text {inc }}$ and $\varphi_{\text {inc }}$ are represented in terms of $\boldsymbol{k}$.

Provided an antenna far field representation that may be decomposed into a $\vartheta$ - and $\varphi$ dependent fraction which are given by $C_{\vartheta, \mathrm{i}}(\vartheta, \varphi)$ and $C_{\varphi, \mathrm{i}}(\vartheta, \varphi)$ in terms of antenna element $i$ and a 2-D polarization invariant probability distribution $p_{\vartheta, \varphi}(\vartheta, \varphi)$ of the AOA with the appropriate normalization given in Waldschmidt and Wiesbeck (2004), Eq. (18) denotes antenna covariance.

$$
\begin{aligned}
& R_{i, j}=\mathrm{K} \int_{0}^{2 \pi} \int_{0}^{\pi}\left[\operatorname{XPR} C_{\vartheta, \mathrm{i}}(\vartheta, \varphi) C_{\vartheta, \mathrm{j}}^{*}(\vartheta, \varphi)\right. \\
& \left.+C_{\varphi, \mathrm{i}}(\vartheta, \varphi) C_{\varphi, \mathrm{j}}^{*}(\vartheta, \varphi)\right] e^{j \boldsymbol{k} \boldsymbol{r}_{i j}} p_{\vartheta, \varphi}(\vartheta, \varphi) \sin \vartheta d \vartheta d \varphi
\end{aligned}
$$

The ratio of the mean incident power in terms of a vertical polarized receive signal to the mean incident power of a horizontal polarized receive signal is denoted by the factor of cross-polarization ratio $X P R$. In Eq. (18), “*” means conjugate operation, $K$ is a proportionality constant.

$$
\begin{aligned}
\sigma_{\mathrm{i}}^{2} & =\mathrm{K} \int_{0}^{2 \pi} \int_{0}^{\pi}\left[\mathrm{XPR}\left|C_{\vartheta, \mathrm{i}}(\vartheta, \varphi)\right|^{2}\right. \\
& \left.+\left|C_{\varphi, \mathrm{i}}(\vartheta, \varphi)\right|^{2}\right] p_{\vartheta, \varphi}(\vartheta, \varphi) \sin \vartheta d \vartheta d \varphi .
\end{aligned}
$$

As the spatial properties of the considered transmit channel strongly influence to the properties of antenna correlation, they have to be inevitably taken into account. An analytical formulation of antenna radiation patterns therefore simplifies the computation of correlation coefficients for diversity analysis. In Leifer (2002) a simplified multipole computational model was published in terms of parallel oriented dipole antennas. It is therefore straightforward to express the vector antenna radiation patterns $\boldsymbol{C}_{\mathrm{i}, \mathrm{j}}(\vartheta, \varphi)$ of antennas $i$ and $j$ in terms of a spherical mode expansion as given in Sect. 3 . Provided the series expansion for the radiation fields as in Eq. (12), the expressions for antenna covariance and variance become a function of modal expansion coefficients $\boldsymbol{a}_{\mathrm{n}}^{\mathrm{m}}$ and $\boldsymbol{b}_{\mathrm{n}}^{\mathrm{m}}$. Equation (20) accounts for the series representation of antenna radiation patterns of antennas $i$ and $j$ by means of spherical eigenmodes and represents a generalized description of antenna covariance in terms of a spherical mode expansion.

$$
\begin{aligned}
& R_{\mathrm{i}, \mathrm{j}}=\mathrm{K} \sum_{n_{i}=0}^{\infty} \sum_{m_{i}=0}^{n_{i}} \sum_{n_{j}=0}^{\infty} \sum_{m_{j}=0}^{n_{j}}\{ \\
& \quad\left(\mathrm{XPR} a_{\mathrm{n}_{\mathrm{i}} \mathrm{m}_{\mathrm{i}}, \vartheta} a_{\mathrm{n}_{\mathrm{j}} \mathrm{m}_{\mathrm{j}}, \vartheta}^{*}+a_{\mathrm{n}_{\mathrm{i}} \mathrm{m}_{\mathrm{i}}, \varphi} a_{\mathrm{n}_{\mathrm{j}} \mathrm{m}_{\mathrm{j}}, \varphi}^{*}\right) I_{\mathrm{n}_{\mathrm{i}} \mathrm{m}_{\mathrm{i}} \mathrm{n}_{\mathrm{j}} \mathrm{m}_{\mathrm{j}}}^{\mathrm{ee}} \\
& +\left(\mathrm{XPR} b_{\mathrm{n}_{\mathrm{i}} \mathrm{m}_{\mathrm{i}}, \vartheta} b_{\mathrm{n}_{\mathrm{j}} \mathrm{m}_{\mathrm{j}}, \vartheta}^{*}+b_{\mathrm{n}_{\mathrm{i}} \mathrm{m}_{\mathrm{i}}, \varphi} b_{\mathrm{n}_{\mathrm{j}} \mathrm{m}_{\mathrm{j}}, \varphi}^{*}\right) I_{\mathrm{n}_{\mathrm{i}} \mathrm{m}_{\mathrm{i}} \mathrm{n}_{\mathrm{j}} \mathrm{m}_{\mathrm{j}}}^{\mathrm{oo}} \\
& +\left(\mathrm{XPR} a_{\mathrm{n}_{\mathrm{i}} \mathrm{m}_{\mathrm{i}}, \vartheta} b_{\mathrm{n}_{\mathrm{j}} \mathrm{m}_{\mathrm{j}}, \vartheta}^{*}+a_{\mathrm{n}_{\mathrm{i}} \mathrm{m}_{\mathrm{i}}, \varphi} b_{\mathrm{n}_{\mathrm{j}} \mathrm{m}_{\mathrm{j}}, \varphi}^{*}\right) I_{\mathrm{n}_{\mathrm{i}} \mathrm{m}_{\mathrm{i}} \mathrm{n}_{\mathrm{j}} \mathrm{m}_{\mathrm{j}}}^{\mathrm{eo}} \\
& \left.+\left(\mathrm{XPR} b_{\mathrm{n}_{\mathrm{i}} \mathrm{m}_{\mathrm{i}}, \vartheta} a_{\mathrm{n}_{\mathrm{j}} \mathrm{m}_{\mathrm{j}}, \vartheta}^{*}+b_{\mathrm{n}_{\mathrm{i}} \mathrm{m}_{\mathrm{i}}, \varphi} a_{\mathrm{n}_{\mathrm{j}} \mathrm{m}_{\mathrm{j}}, \varphi}^{*}\right) I_{\mathrm{n}_{\mathrm{i}} \mathrm{m}_{\mathrm{i}} \mathrm{n}_{\mathrm{j}} \mathrm{m}_{\mathrm{j}}}^{\mathrm{oe}}\right\}
\end{aligned}
$$

As can be seen in (20), covariance computation requires a fourfold summation of 2-D coupling integrals $I_{\mathrm{n}_{\mathrm{i}} \mathrm{m}_{\mathrm{i}} \mathrm{n}_{\mathrm{j}} \mathrm{m}_{\mathrm{j}}}^{\mathrm{e} \text { e, }}$ that are weighed by the respective expansion coefficients. Therefore an evaluation of Eq. (20) might become quite time consuming with the advantage to derive an analytical formulation of the correlation properties in multielement antenna configurations. The coupling integrals are given in terms of a product of two normalized spherical harmonics $Y_{\mathrm{nm}}^{\mathrm{e}, \mathrm{o}}(\vartheta, \varphi)$ 


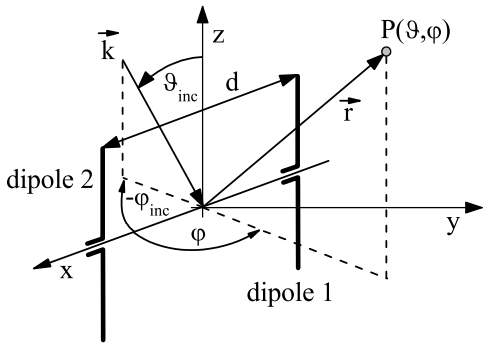

Fig. 3. Configuration of two parallel oriented $\lambda / 2$ dipoles with a relative displacement of $d$ along the $x$-axis.

with the angular distribution of incoming waves, $p_{\vartheta, \varphi}(\vartheta, \varphi)$ as shown in Eq. (21).

$$
\begin{aligned}
I_{\mathrm{n}_{\mathrm{i}} \mathrm{m}_{\mathrm{i}} \mathrm{n}_{\mathrm{j}} \mathrm{m}_{\mathrm{j}}}^{\mathrm{e}, \mathrm{e}, \mathrm{s}}= & \int_{0}^{2 \pi} \int_{0}^{\pi} Y_{\mathrm{n}_{\mathrm{i}} \mathrm{m}_{\mathrm{i}}}^{\mathrm{e}, \mathrm{o}}(\vartheta, \varphi) Y_{\mathrm{n}_{\mathrm{j}} \mathrm{m}_{\mathrm{j}}}^{\mathrm{e}, \mathrm{o}}(\vartheta, \varphi) \\
& \cdot e^{j \boldsymbol{k} \boldsymbol{r}_{i j}} p_{\vartheta, \varphi}(\vartheta, \varphi) \sin \vartheta d \vartheta d \varphi
\end{aligned}
$$

Using the orthogonality relation as in Werner and Mittra (2000) for spherical harmonics, Eq. (20) may be simplified with respect to a certain symmetry of the AOA distribution $p_{\vartheta, \varphi}(\vartheta, \varphi)$ and the antenna array topology.

Applying the spherical wave expansion to the general formulation of variances as in Eq. (19) for antenna element $v$, $\nu=1,2$ yields:

$$
\begin{gathered}
\sigma_{v}^{2}=\mathrm{K} \int_{0}^{2 \pi} \int_{0}^{\pi}\left\{\mathrm{XPR} \mid \sum_{n_{v}=0}^{\infty} \sum_{m_{v}=0}^{n_{v}} a_{\mathrm{n}_{v} \mathrm{~m}_{v}, \vartheta} Y_{\mathrm{n}_{v} \mathrm{~m}_{v}}^{\mathrm{e}}\right. \\
+\left.b_{\mathrm{n}_{v} \mathrm{~m}_{v}, \vartheta} Y_{\mathrm{n}_{v} \mathrm{~m}_{v}}^{\mathrm{o}}\right|^{2}+\mid \sum_{n_{\nu}=0}^{\infty} \sum_{m_{v}=0}^{n_{v}} a_{\mathrm{n}_{v} \mathrm{~m}_{v}, \varphi} Y_{\mathrm{n}_{v} \mathrm{~m}_{v}}^{\mathrm{e}} \\
\left.+\left.b_{\mathrm{n}_{v} \mathrm{~m}_{v}, \varphi} Y_{\mathrm{n}_{v} \mathrm{~m}_{v}}^{\mathrm{o}}\right|^{2}\right\} p_{\vartheta, \varphi}(\vartheta, \varphi) \sin \vartheta d \vartheta d \varphi .
\end{gathered}
$$

\section{Analysis of Two-Element Dipole Array}

The correlation analysis of an arrangement of two parallel oriented, resonant $\lambda / 2$ dipoles in free space depicted in Fig. 3 will be accomplished in this section. In order to compare the results from the customary computation of antenna correlation by an evaluation of the twofold Riemann sum in Eqs. (18) and (19) with the method based upon a spherical mode expansion of antenna radiation patterns as given in Sect. 4 the patterns are evaluated in terms of a spherical mode expansion delimited by $N$. In terms of the correlation analysis of the two-element dipole configuration two different cases have to be considered. The first case assumes both dipole antennas isolated from each other, so that the effect of electromagnetic interaction is neglected by definition. The assumption justifies a modification of the field expansion applying the array factor of the antenna configuration into the correlation analysis. The results of this study are summarized in Sect. 5.1. In the second approach electromagnetic coupling between the dipole antennas is taken into account.

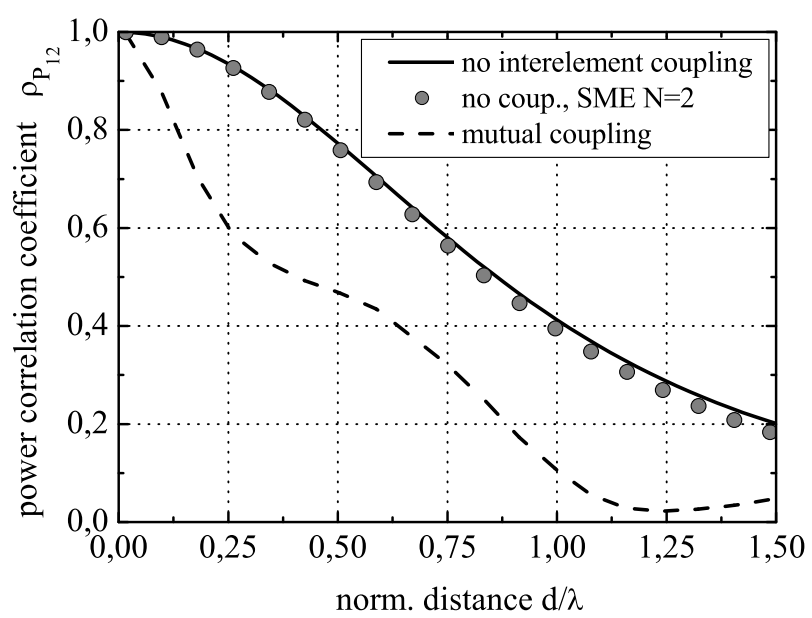

Fig. 4. Power correlation of isolated- and coupled dipole antennas for an angular spread of $\sigma_{\varphi}=10^{\circ}$.

Section 5.2 provides the results for the coupling-case of the dipole array.

\subsection{Analysis of isolated Dipoles}

In terms of a configuration of identical isolated antenna elements, the representation for the total radiation pattern can be simplified with respect to the appropriate array factor $G(\vartheta, \varphi)$ of the antenna configuration. Therefore the computation of antenna correlation simplifying requires the spherical mode expansion of the radiation fields of the isolated antenna element that is identical for all elements within the arrangement. The consideration of an array of identical isolated antenna elements therefore leads to an effective reduction of the computational resources for correlation analysis. For convenience, the AOA distribution will be restricted to the $x y$-plane and hence be given in terms of a Laplacian distribution as in Svantesson (2002) that is properly described by a mean value $m_{\varphi}$ and the respective spread $\sigma_{\varphi}$. All simulations are performed using a mean value of $m_{\varphi}=90^{\circ}$ using the coordinate system depicted in Fig. 3. Three different values of angular spread $\sigma_{\varphi}$ are considered. Figure 4 depicts antenna power correlation coefficient with respect to the normalized interelement distance $d / \lambda$ and a narrow angular spread $\sigma_{\varphi}=10^{\circ}$. In order to address to the influences of electromagnetic coupling on antenna correlation, the dashed lines in Fig. 4 represent the correlation coefficient in terms of coupled dipole antennas derived from the customary evaluation of Eqs. (18) and (19). The results derived from a spherical wave expansion of the radiation pattern of the isolated dipole antennas are given as circles. The series representation in (Eq. 12) is restricted with respect to $N=2$ leading to a maximum of 12 complex vector coefficients in the field expansion. Comparing the processes of antenna correlation for the uncoupled arrangement and the case of an electromagnetically coupled antenna configuration, interelement coupling leads to a decrease of antenna correlation for 


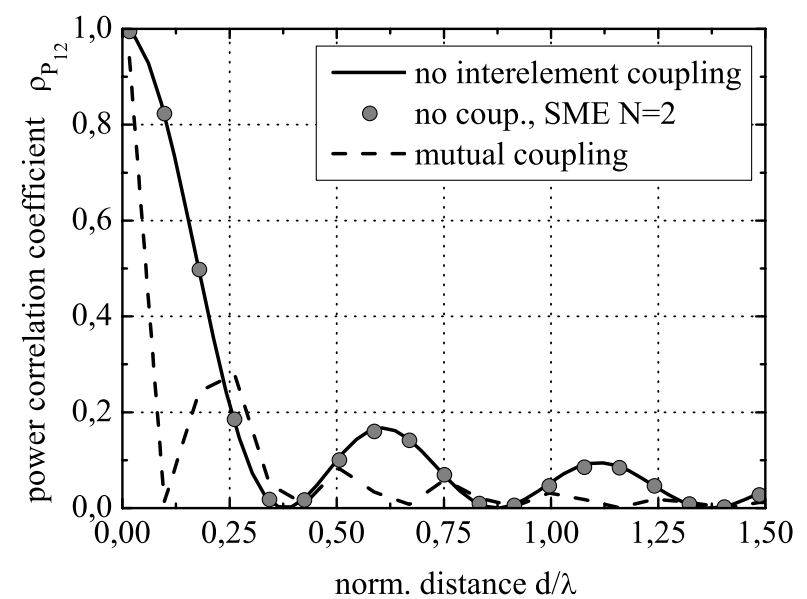

Fig. 5. Power correlation of isolated- and coupled dipole antennas in terms of equally distributed AOAs in the horizontal plane.

all interelement coupling distances. This observation is in accordance to Tsunekawa and Kagoshima (1990) as well as Leifer (2002). The degradation of antenna correlation is due to the effect of pattern diversity that can only be contributed of an arrangement of electromagnetically coupled antenna elements. Antenna correlation of the electromagnetically decoupled dipole antennas is restricted to the occurrence of spatial diversity due to antenna separation $\boldsymbol{r}_{\mathbf{i j}}$. The results for the correlation analysis based upon the spherical mode expansion of the radiation field matches very well with the results derived from customary correlation analysis, unless only 12 vector coefficients are involved in the field expansion. This may be explained in terms of the convergence criterion given by Narasimhan et al. (1985) that claims modal indices $N_{\min }=k a$, with $a=\lambda / 2$ with respect to the configuration of $\lambda / 2$-resonant, uncoupled dipole antennas. Therefore the convergence criterion requires integer indices of $N_{\min }=2$. It has to be clarified, that the processes of antenna correlation derived from the customary numerical analysis of Eq. (17) using Eqs. (18) and (19) may only be considered to be approximative solutions. As a result of numerical simulation applying the FEM, antenna far field representation may not be given in terms of an analytical formulation. The results were obtained by applying an equiangular grid on the far field sphere, where the radiation fields were calculated. Throughout this paper equal grid distances in the directions of azimuth and elevation, $\Delta \varphi=3^{\circ}$ and $\Delta \vartheta=3^{\circ}$ were applied.

Figure 5 considers the case of an equal angle of arrival distribution in the $x y$-plane. Neglecting the effects of interelement coupling between the dipole antennas, the observed antenna correlation corresponds to the well known result for the power correlation of Jakes (1974) between antennas $i$ and $j$ using the Bessel function of 0 . order, with $\rho_{P_{\mathrm{i}, \mathrm{j}}}=J_{0}^{2}(k d)$. As observed from Fig. 4 mutual coupling between adjacent antenna elements also leads to a decrease of power correlation (e.g. Fig. 5) due to pattern diversity gain, especially for small antenna displacements. The results of

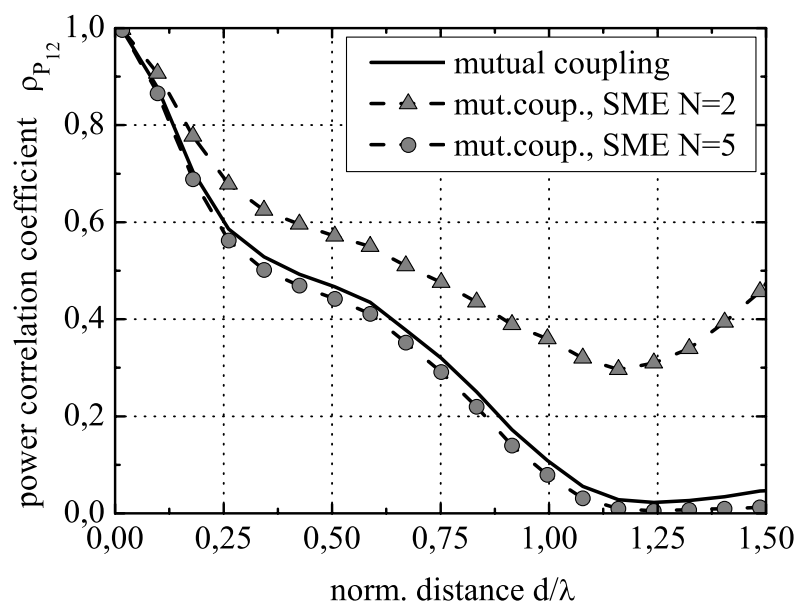

Fig. 6. Power correlation of coupled dipole antennas for an angular spread of $\sigma_{\varphi}=10^{\circ}$ and different degrees of SME.

correlation analysis with respect to the SME of the radiation fields for $N=2$ again reveals a good agreement to the customary computation technique.

\subsection{Analysis of coupled Dipoles}

From Sect. 5.1 it can be concluded, that mutual coupling between adjacent elements within a multielement antenna arrangement may result in an improvement of diversity performance. This increase of diversity gain is a result of a degradation of antenna radiation patterns losing their axial symmetry. Therefore the convergence of antenna radiation pattern analysis is influenced by the appearance of mutual coupling causing higher order modes in the field expansion. As a result, a larger number of vector expansion coefficients has to be taken into account to guarantee an accurate approximation of antenna radiation behavior.

This effect can be derived from Fig. 6 that shows the results of antenna correlation for an angular spread of $\sigma_{\varphi}=10^{\circ}$. The computation of interelement antenna correlation is based upon field expansions dealing with summation limits of $N=2$ and $N=5$ in accordance with Eq. (12). The differences in simulation results between the customary computation technique by sampling the far field sphere and the series expansion in terms of spherical harmonics are minimized with respect to an increase of $N$. This is due to the violation of the convergence criterion $N_{\min }=k a$ for low degrees of the field expansion. With an increase of the normalized interelement distance $d / \lambda$ of the coupled dipole configuration, the effective antenna aperture increases. Therefore an enhanced number of spherical modes has to be taken into account, to ensure sufficient field accuracy. E.g. for a given interelement spacing of $d / \lambda=1.5$, modal indices of degree $N_{\min }=4$ should be considered in the field approach.

Figure 7 represents the results of antenna correlation for an equally distributed AOA in the horizontal plane. As expected the results show the same bias. Due to the increase 


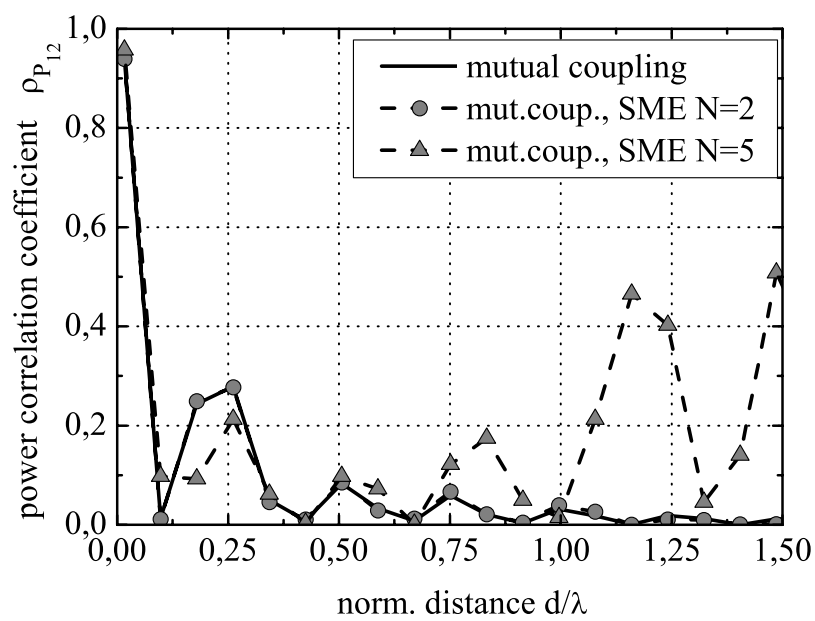

Fig. 7. Power correlation of coupled dipole antennas in terms of equally distributed AOAs in the horizontal plane and different degrees of SME.

of effective antenna aperture and the degradation of antenna radiation behavior from the axial symmetry of the isolated dipole antenna, a larger series limit $N$ is required to account for a sufficient approximation of antenna radiation fields. With an increase of the number of vector expansion coefficients in Eq. (12) the curves of antenna correlation tend to converge.

\section{Analysis of Frequency-Independent Planar Antennas}

Compact multimode antennas as the biconical antenna that has been reported by Svantesson (2002) or the planar multiterminal spiral- and sinuous antennas reported by Waldschmidt and Wiesbeck (2004) seem to be promising for the integration in MIMO transmission systems with a combination of polarization-, pattern- and modal diversity. In this section the four-arm log.-per. trapezoid antenna, that was reported by Klemp et al. (2004a) and a miniaturized derivative of this antenna type, the radial modulated four-arm log.-per. trapezoid antenna reported by Klemp et al. (2004b) will be analyzed with respect to their property of polarization diversity. The antennas adhere to the principle of self-complement and therefore provide large operational bandwidths. Both antenna types are depicted in Fig. 8. The ability of a good polarization diversity behavior is based on the degeneration of two spatially orthogonal eigenfield distributions, that are supplied by the symmetrical four-arm antennas in Fig. 8. In the ideal case the four arms of the log.-per. antenna structures can be decomposed into two orthogonal arm pairs that represent two decoupled transmit channels with orthogonal linear polarization leading to uncorrelated output voltages at the feeding bridge of the antenna structure.

For the evaluation of polarization diversity performance a 2-D probability distribution of the angle of arrival is presumed. In analogy to the receive diversity case of the dipole

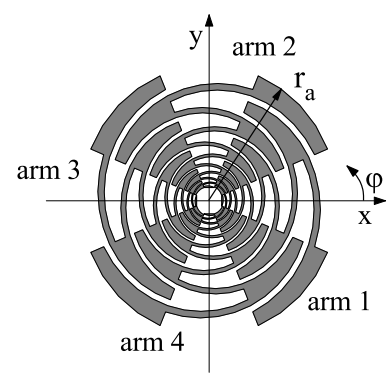

a) four-arm trapezoid antenna

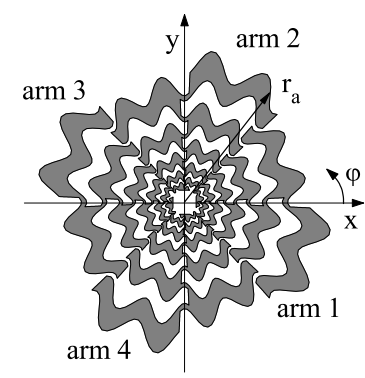

b) radial modulated trapezoid ant.
Fig. 8. Trapezoidal antenna geometries for the realization of frequency-independent diversity antennas.

antenna array, the azimuthal dependence of AOA is modeled using a Laplacian distribution. In terms of the elevational dependence, a Gauss distribution is assumed in accordance with Waldschmidt and Wiesbeck (2004). Mean value and angular spread in the elevation are given as $m_{\vartheta}=45^{\circ}, \sigma_{\vartheta}=15^{\circ}$. The Laplace distribution is determined by a mean value of $m_{\varphi}=10^{\circ}$ and a spread of $\sigma_{\varphi}=20^{\circ}$.

Figure 9 shows the result of power correlation coefficient $\rho_{\mathrm{P}_{\mathrm{xy}}}$ in terms of polarization diversity performance with equal mean branch power in each polarization component ( $x$-polarization or $y$-pol.) in the specified frequency range from $1 \mathrm{GHz}$ to $6 \mathrm{GHz}$. Due to the high degree of cross polarization discrimination of each pair of opposite antenna arms, a low degree of power correlation can be observed in the entire frequency range of operation. $\rho_{P}$ always remains below 0.12 . The results of an ordinary computation of power correlation are compared to the results of a spherical mode expansion $(N=5)$ for the trapezoid antenna leading to a good agreement. The low degree of correlation can be justified by using the results derived from spherical antenna near field measurement at the distinct frequency positions $f_{1}=1.8 \mathrm{GHz}, f_{2}=2.45 \mathrm{GHz}$ and $f_{3}=5.85 \mathrm{GHz}$. Based upon the measurement results with an angular resolution of $1^{\circ}$ in $\vartheta$ - and $\varphi$-direction the power correlation coefficient was computed using the twofold Riemann approximation of Eqs. (17) to (19). As for the miniaturized derivative of the four-arm trapezoid antenna depicted in Figs. 8b, 10 represents the results of power correlation against operating frequency. Results derived from an approach of Eq. (17) by taking the equivalent Riemann sums of Eqs. (18) and (19) as well as the evaluation of power correlation by spherical mode expansion of the individual radiation patterns yield a good agreement. Numerical data of power correlation can be verified very well by results from spherical near field measurement of the miniaturized trapezoid antennas at the respective frequency positions $f_{1}$ to $f_{3}$ as shown in Fig. 10.

\section{Conclusions}

In this paper, a generalized approach for the computation of power correlation in multielement antenna arrangements was 


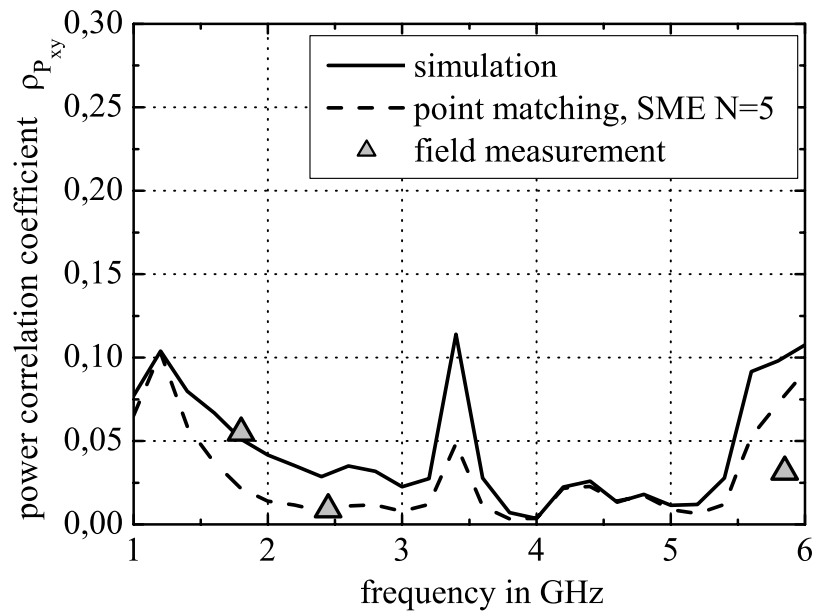

Fig. 9. Power correlation for log.-per. trapezoid antenna. Simulation and measurement results.

presented, which is based upon the expansion of antenna radiation fields in terms of spherical harmonics. Therefore antenna radiation behavior was considered in receive-mode. The spatial properties of a multipath propagation scenario were modeled using 2-D probability density distributions. In terms of a finite series representation of antenna radiation fields, an analytical investigation of antenna correlation properties can be accomplished. An analytical formulation of antenna power correlation was presented involving elementary 2-D coupling integrals over a product of spherical harmonics, that are weighed by the vector series expansion coefficients of the corresponding antenna radiation patterns. Due to the fact that the contributions of elementary coupling integrals remain fixed for a given array topology and propagation environment, the amount of antenna correlation only depends on the expansion coefficients of their radiation pattern and therefore from the current distribution on the corresponding metalization surfaces. Hence the presented analysis provides a major simplification in order to derive the respective correlation properties of individual antenna elements in a multipath propagation scenario. Furthermore the analysis may serve as an initial point for the optimization of antenna radiation properties with respect to low interelement correlations by adjusting the modal content of the respective radiation fields.

The presented correlation analysis was applied on a configuration involving two parallel resonant $\lambda / 2$ dipole antennas in receive mode differentiating between isolated and electromagnetically coupled antennas. The results of customary correlation analysis and SME based computation of antenna correlation agree very well. Likewise the two different approaches in correlation analysis were adopted to the characterization of two wideband log.-per. planar antennas in polarization diverse receive mode. The results also reveal a very good agreement and could be verified very well by results derived from spherical antenna near field measurement.

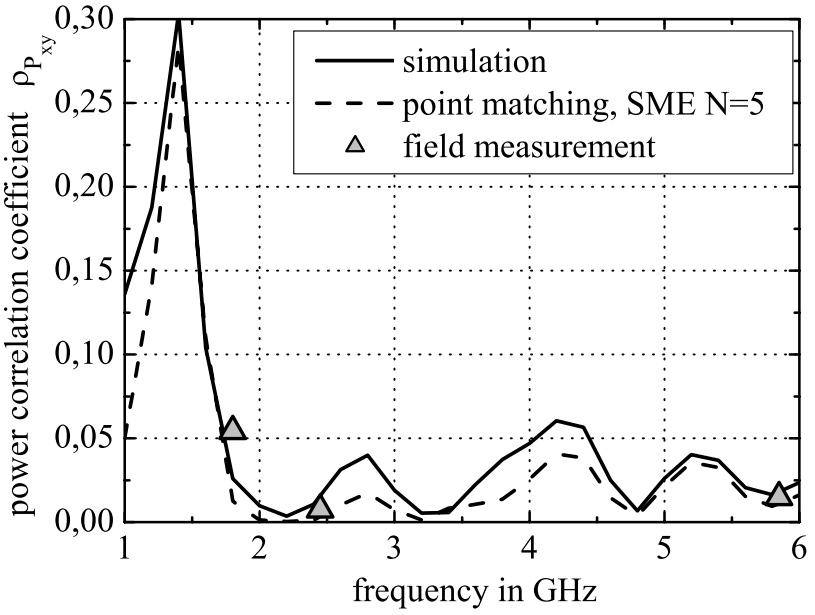

Fig. 10. Power correlation for miniaturized log.-per. trapezoid antenna. Simulation and measurement results.

The self-complementary log.-per. four-arm trapezoid antennas may be considered for an implementation in a transmission system with wideband polarization diversity reception and seem promising for the integration in MIMO transmission systems with multimode operation due to the multi-port antenna concept and low correlations between the different operational modes.

\section{References}

Abramovitz, M. and Stegun, I. A.: Handbook of Mathematical Functions, Dover Publications, New York, 1972.

Adane, Y., Gati, A., Wong, M.-F., Dale, C., Wiart, J., and Hanna, V. F.: Optimal Modeling of Real Radio Base Station Antennas for Human Exposure Assessment Using Spherical-Mode Decomposition, IEEE Antennas and Wireless Propagation Letters, 1, 215-218, 2002.

Bruning, J. H. and Lo, Y. T.: Multiple Scattering of EM Waves by Spheres Part I-Multipole Expansion and Ray-Optical Solutions, IEEE Transactions on Antennas and Propagation, 19, 378-390, 1971.

Chen, Y. and Simpson, T.: Radiation Pattern Analysis of Arbitrary Wire Antennas Using Spherical Mode Expansions with Vector Coefficients, IEEE Transactions on Antennas and Propagation, 39, 1716-1721, 1991a.

Chen, Y. and Simpson, T. L.: Compact Representation of Radiation Patterns using Vector Spherical Mode Expansions, Antennas and Propagation Society International Symposium, 24-28 June 1991, 3, 1632-1634, $1991 b$.

Chen, Y., Simpson, T. L., and Ho, T. Q.: Highly efficient technique for solving radiation and scattering problems, IEE ProceedingsH, 139, 7-10, 1992.

Derneryd, A. and Kristensson, G.: Antenna signal correlation and its relation to the impedance matrix, Electronics Letters, 40, 401402, 2004.

Hafner, C.: The Generalized Multipole Technique for Computational Electromagnetics, Artech House, Inc., Boston, London, 1990. 
Jakes, W. C.: Microwave Mobile Communications, IEEE Press, Inc., New York, 1974.

Kildal, P.-S. and Rosengren, K.: Electromagnetic Analysis of Effective and Apparent Diversity Gain of Two Parallel Dipoles, IEEE Antennas and Wireless Propagation Letters, 2, 9-13, 2003.

Klemp, O., Schultz, M., and Eul, H.: A Planar Broadband Antenna for Applications in WLAN and 3G, International Symposium on Signals, Systems and Electronics ISSSE, Linz, Austria, August 10-13, 2004a.

Klemp, O., Schultz, M., and Eul, H.: Miniaturization Techniques For Logarithmically-Periodic Planar Antennas, IEEE International Symposium on Personal Indoor and Mobile Communications PIMRC, Barcelona, Spain, September 5-9, 2004b.

Leifer, M. C.: Signal Correlations in Coupled Cell and MIMO antennas, Antennas and Propagation Society International Symposium, 16-22 June 2002, 3, 194-197, 2002.

Ludwig, A. C.: Near-Field Far-Field Transformations Using Spherical-Wave Expansions, IEEE Transactions on Antennas and Propagation, 19, 214-220, 1971.

Narasimhan, M. S., Christopher, S., and Varadarangan, K.: Modal Behavior of Spherical Waves from a Source of EM Radiation with Application to Spherical Scanning, IEEE Transactions on Antennas and Propagation, 33, 350-354, 1985.
Potter, P. D.: Application of Spherical Wave Therory to Cassegrainian-Fed Paraboloids, IEEE Transactions on Antennas and Propagation, 15, 727-736, 1967.

Stratton, J. A.: Electromagnetic Theory, McGraw Hill, New York, 1941.

Svantesson, T.: Correlation and Channel Capacity of MIMO Systems Employing Multimode Antennas, IEEE Transactions on Vehicular Technology, 51, 1304-1312, 2002.

Tsunekawa, K. and Kagoshima, K.: Analysis of a Correlation Coefficient of Built-In Diversity Antennas for a Portable Telephone, Antennas and Propagation Society International Symposium, 7 11 May 1990, 1, 543-546, 1990.

Waldschmidt, C. and Wiesbeck, W.: Compact Wide-Band Multimode Antennas for MIMO and Diversity, IEEE Transactions on Antennas and Propagation, 52, 1963-1969, 2004.

Werner, D. H. and Mittra, R.: Frontiers in Electromagnetics, IEEE Press Series on Microwave Technology and RF, New York, 2000.

Witte, E. D., Griffiths, H. D., and Brennan, P. V.: Phase mode processing for spherical antenna arrays, Electronics Letters, 39, 1430-1431, 2003. 